\section{OP0105 \\ ZCCHC6 DELETION DOWNREGULATES IL-6 AND REDUCES THE SEVERITY OF EXPERIMENTAL OSTEOARTHRITIS IN MICE}

M.Y. Ansari, M.N. Khan, I. Ahmad, T.M. Haqqi. Anatomy and Neurobiology, Northeast Ohio Medical University, Rootstown, USA

Background: Osteoarthritis $(\mathrm{OA})$ is the most common joint disease in elderly and is a major cause of disability. Currently no disease modifying agents exist to treat OA. ZCCHC6 (zinc-finger CCHC-domain containing protein 6) is a member of family of terminal uridylyltransferases (TUTs) and is known to carry out template independent addition of Uridine at the 3' ends of miRNAs. ZCCHC6 has been implicated in miRNA-mediated regulation of cytokine gene expression; however, the role of $\mathrm{ZCCHC} 6$ in the pathogenesis of $\mathrm{OA}$ is unknown.

Objectives: The aim of this study was to investigate the effect of ZCCHC6 deletion on proinflammatory cytokine, IL-6, expression in human and mouse chondrocytes and to study the role of ZCCHC6 in OA pathogenesis in vivo using Zcchc6-/mice.

Methods: Human or mouse chondrocytes were prepared by sequential enzymatic digestion and treated with recombinant human or mouse IL-1 $\beta$ to mimic OA pathogenic conditions. Cytokine profiling was done using qPCR array and multiplex assay. IL-6 expression in chondrocytes was analysed by qPCR and immunoblotting/ELISA. IL-6 mRNA stability was determined by Actinomycin-D chase experiments. The 3'-uridylation of miRNAs was analysed by deep sequencing of small RNAs using Illumina MiSeq. ZCCHC6 and IL-6 expression in OA cartilage was analysed by immunohistochemistry. For in vivo studies, OA was induced by surgical destabilisation of medial meniscus (DMM) in the knee joints of mice. The severity of OA was assessed by safranin O/fast green staining followed by OARSI scoring.

Results: ZCCHC6 expression was upregulated in OA cartilage. Cytokine expression profiling in ZCCHC6 depleted human and Zcchc6-/- mouse chondrocytes revealed IL- 6 as a major target of ZCCHC6. Depletion of ZCCHC6 expression in human chondrocytes resulted in decreased expression of IL-6 at mRNA and protein levels. ZCCHC6 depletion suppressed IL- $1 \beta$ induced expression of IL-6. Overexpression of ZCCHC6 in human chondrocytes resulted in increased expression of IL-6 in the presence or absence of IL-1ß. Chondrocytes from Zcchc6-/mice also showed decreased levels of IL-6 expression compared to Zcchc6+/+ Furthermore, overexpression of mouse Zcchc6 in Zcchc6-/- chondrocytes rescued IL-6 expression. IL-6 mRNA half-life was significantly reduced in Zcchc6-/mouse chondrocytes and ZCCHC6 depleted human chondrocytes. Deep sequencing of small RNAs in ZCCHC6 depleted human chondrocytes showed reduced 3'-uridylation of IL-6 targeting miRNAs miR-26a/26b. Human chondrocytes transfected with miR-26a/26b mimic suppressed IL-6 expression, however, miR-26b mimic with additional 'UU' failed to suppress IL-6 expression. Zcchc6-/mice expressed low levels of MMP13 and showed lesser matrix degradation in the joints with DMM surgery. Zcchc6-/- mice developed less severe OA as determined by safranin O/fast green staining followed by OARSI scoring. Synovitis was also decreased in the Zcchc6-/- mice DMM joints in comparison to Zcchc6+/ + DMM joints.

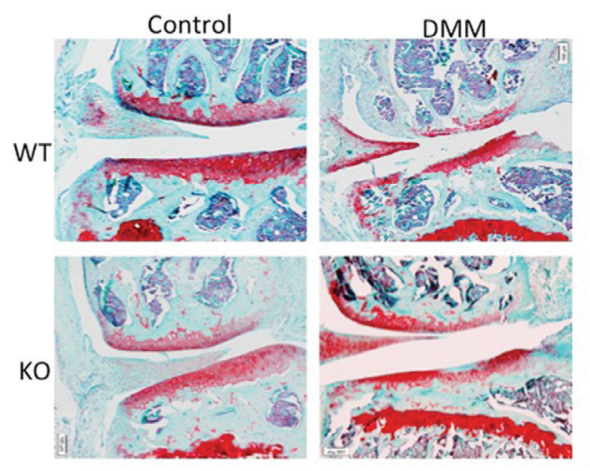

Conclusions: Our data demonstrate that $\mathrm{ZCCHC} 6$ is upregulated in $\mathrm{OA}$ cartilage and regulates IL-6 expression via miR-26b 3'-uridylation. These data identify a previously unknown function of ZCCHC6 in OA pathogenesis and identifies a potential therapeutic target for the management of $\mathrm{OA}$.

Acknowledgements: This project is supported by NIH grant (RO1-AT007373, RO1-AT-005520, RO1-AR-067056) and funds from Northeast Ohio Medical University.

Disclosure of Interest: None declared

DOI: 10.1136/annrheumdis-2018-eular.2071
WEDNESDAY, 13 JUNE 2018

\section{Delay in treatment and the role of health professionals}

\section{OP0106 WHO IS NOT REACHING REMISSION IN EARLY RA AND WHY? PREDICTORS FOR PERSISTENT DISEASE ACTIVITY IN THE FIRST YEAR DIFFER IN MEN AND WOMEN AND ARE RELATED TO LIFESTYLE AND TREATMENT}

S.J Bartlett ${ }^{1,2}$, O. Schieir ${ }^{3}$, M.-F. Valois ${ }^{4}$, C. Hitchon $^{5}$, G. Boire ${ }^{6}$, B. Haraoui ${ }^{7}$, C. Thorne ${ }^{8}$, D. Tin ${ }^{8}$, J. Pope ${ }^{9}$, E.C. Keystone ${ }^{3,10}$, V.P. Bykerk ${ }^{3,11}$, on behalf of on behalf of CATCH (Canadian Early Arthritis Cohort) Investigators. ${ }^{1}$ Medicine/ClinEpi and Rheumatology, McGill University, Montreal, QC, Canada; ${ }^{2}$ Medicine Rheumatology, Johns Hopkins, Baltimore, USA; ${ }^{3}$ University of Toronto, Toronto, ON; ${ }^{4}$ Clinical Epidemiology, McGill University, Montreal, QC; ${ }^{5}$ Rheumatology, University of Manitoba, Winnipeg, MB; ${ }^{6}$ Rheumatologie, Universite de Sherbrooke, Sherbrooke, QC; ${ }^{7}$ Institute de Rheumatologie, Montreal, QC; ${ }^{8}$ Southlake Regional Health Centre, Newmarket, ON; ${ }^{9}$ Western University, London, ON; ${ }^{10}$ Sinai Health System, Toronto, ON, Canada; ${ }^{11}$ Hospital for Special Surgery, New York, NY, USA

Background: Although early identification and aggressive treatment of RA improves outcomes, we have shown that $45 \%$ of early RA participants receiving guideline-based care do not achieve remission in the first year. Moreover, fewer women reached remission than men.

Objectives: To compare predictors of persistent disease activity (LDA/MDA/ $H A D$ ) in the 1 st year of RA treatment in men and women.

Methods: Sample included adults in CATCH (Canadian Early Arthritis Cohort) from 2007-2016 with active disease at baseline and $>12 \mathrm{~m}$ F/U. Standardised visits included clinical assessments, questionnaires, and lab tests. Logistic regression with backward selection was used to identify predictors of failing to achieve remission (DAS28 <2.6) by 12 months among baseline sociodemographic and RA characteristics and patient reported outcomes.

Results: The sample included 1628 adults with classifiable RA, who were mostly female (72\%) with a mean (SD) age of 55, (15) with 2 (2) comorbidities, and symptom duration of 6 (3) months. At enrollment, $95 \%$ had active disease (DAS28 MDA $(42 \%)$; HDA (53\%)), most were initially treated with csDMARDS (any $92 \%$; MTX $75 \%$ ). $46 \%$ of women and $38 \%$ of men did not reach remission by 12 months. Among women, multivariable results showed obesity more than doubled the likelihood of not achieving remission; other key predictors were minority status, lower education, and higher TJC and fatigue scores at baseline (table 1). In men, current smoking was associated with a 3.5 greater odds of not achieving remission in the first year; other predictors included older age, and higher pain. Not using MTX increased the likelihood of not achieving remission in women by $28 \%$ and men by $45 \%$. Longer symptom duration and higher ESR were associated with not achieving remission in all. Factors not related to persistent disease activity included family history of RA, RF/ACPA status, erosions, SJC, HAQ and depressive symptoms at baseline.

Abstract OP0106 - Table 1

\begin{tabular}{|c|c|c|}
\hline & Women & Men \\
\hline $\mathrm{N}$ & 1179 & 449 \\
\hline \multicolumn{3}{|l|}{ Sociodemographics } \\
\hline Age (each 10 years) & $1.04(0.95,1.14)$ & $1.48(1.22,1.79)$ \\
\hline Minority race & $1.46(1.07,2.00)$ & Not selected \\
\hline Education = high school & $1.41(1.09,1.82)$ & Not selected \\
\hline Comorbidities & $1.12(1.05,1.21)$ & Not selected \\
\hline Obese $\left(\mathrm{BMI} \geq 30 \mathrm{~kg} / \mathrm{m}^{2}\right)$ & $2.06(1.49,2.83)$ & $1.65(0.95,2.85)$ \\
\hline Current smoking & Not selected & $3.45(2.06,5.77)$ \\
\hline \multicolumn{3}{|l|}{ RA Characteristics } \\
\hline Symptom duration (month) & $1.10(1.05,1.14)$ & $1.15(1.07,1.24)$ \\
\hline Family history of RA & Not selected & $1.62(0.95,2.77)$ \\
\hline $\mathrm{RF}+$ or $\mathrm{ACPA}+$ & $1.23(0.87,1.75)$ & Not selected \\
\hline Erosions & Not selected & Not selected \\
\hline MTX Use & $0.72(0.54,0.95)$ & $0.55(0.33,0.90)$ \\
\hline Oral Steroids ( $\mathrm{Y}$ vs $\mathrm{N}$ ) & $0.80(0.60,1.07)$ & $1.45(0.92,2.27)$ \\
\hline Parenteral Steroids ( $\mathrm{Y}$ v N $)$ & Not selected & Not selected \\
\hline $\operatorname{ESR}(\mathrm{mm} / \mathrm{h})$ & $1.01(1.01,1.02)$ & $1.02(1.01,1.03)$ \\
\hline Swollen Joint Count (28) & Not selected & Not selected \\
\hline Tender Joint Count (28) & $1.03(1.01,1.06)$ & Not selected \\
\hline \multicolumn{3}{|l|}{ Patient Reported Outcomes } \\
\hline High depressive symptoms & Not selected & Not selected \\
\hline Pain (0-10) & Not selected & $1.11(1.03,1.20)$ \\
\hline HAQ-DI (0-3) & Not selected & Not selected \\
\hline Fatigue $(0-10)$ & $1.05(1.00,1.09)$ & Not selected \\
\hline \multicolumn{3}{|l|}{ Fit Statistics } \\
\hline AIC & 1519 & 535 \\
\hline $\mathrm{BIC}$ & 1601 & 581 \\
\hline Area under the curve & 0.69 & 0.74 \\
\hline
\end{tabular}

Conclusions: In this large pan-Canadian cohort of early RA patients receiving guideline-based arthritis care, obesity in women and current smoking in men were the strongest predictors of not achieving remission in the first 12 months followed by non-use of MTX, higher baseline inflammation and longer symptom duration. Additional poor prognostic indicators in women included minority status, lower 
education, and higher fatigue, whereas older age and greater pain were associated with persistent disease activity in men. Smoking cessation in men and weight reduction in women, and optimising MTX use may facilitate rapid reduction of inflammation, an essential goal of treatment in early RA.

Acknowledgements: Sponsors: Amgen \& Pfizer-Founding sponsors 2007+; UCB, AbbVie 2011+; Medexus 2013+; Eli Lilly, Sanofi- Genzyme 2016+; Merck 2017+; BMS 2011-2014, Hoffmann-LaRoche, Janssen 2011-2016

Disclosure of Interest: None declared

DOI: 10.1136/annrheumdis-2018-eular.5848

\section{THURSDAY, 14 JUNE 2018}

\section{Fat and fatty acids: targets for therapy?}

\section{OP0107 ADIPOCYTOKINES IMBALANCE IS ASSOCIATED WITH VASCULAR DAMAGE IN SYSTEMIC SCLEROSIS}

S. Bosello ${ }^{1}$, E. De Lorenzis ${ }^{1}$, G. Canestrari ${ }^{1}$, G. Natalello ${ }^{1}$, C. Di Mario ${ }^{1}$, L. Gigante ${ }^{1}$, A. Barini ${ }^{2}$, A. Barini ${ }^{2}$, L. Verardi ${ }^{1}$, B. Tolusso ${ }^{3}$, G. Ferraccioli ${ }^{1}$, E. Gremese ${ }^{1} .{ }^{1}$ Rheumatology Unit, ${ }^{2}$ Department of Laboratory Medicine, Fondazione Policlinico Universitario A. Gemelli - Catholic University of the Sacred Heart, Rome; ${ }^{3}$ Institute of Rheumatology, Catholic University of the Sacred Heart Fondazione Policlinico Universitario A. Gemelli, Roma, Italy

Background: Adipocytokines are implicated in the development of fibrosis, vasculopathy and immune abnormalities through a variety of biological effects, but their role in systemic sclerosis (SSc) is not fully investigated. Chemerin is implicated in chemotaxis of immune cells, in promoting angiogenesis and it is involved in inflammation. Adiponectin (APN) has metabolic actions and anti-inflammatory properties, while Leptin (LEP) mediates actions in endothelial cells, such as angiogenesis, vasodilation, NO production and upregulates various mediators of vascular inflammation.

Objectives: In this study we investigated Chemerin, LEP and APN levels in SSc patients according to disease subtypes and clinical characteristics.

Methods: Chemerin, LEP and APN levels were evaluated in 100 SSc patients and in sex, age and BMI matched healthy controls. Clinical and demographical characteristics were available for all patients.

Results: Chemerin, APN and LEP levels were lower in SSc patients compared to healthy controls (Chemerin: $58.7 \pm 27.6 \mathrm{ng} / \mathrm{ml}$ vs $74.0 \pm 29.0 \mathrm{ng} / \mathrm{ml}, \mathrm{p}=0.004$; LEP: $19.6 \pm 18.3 \mathrm{ng} / \mathrm{ml}$ vs $28.5 \pm 23.8 \mathrm{ng} / \mathrm{ml}, \mathrm{p}=0.03$, APN: $6.5 \pm 3.9 \mu \mathrm{g} / \mathrm{ml}$ vs 12.8 $\pm 6.0 \mu \mathrm{g} / \mathrm{ml}, \mathrm{p}<0.001$ )

Chemerin levels were lower in patients with anti-topoisomerase antibodies $(50.2$ $\pm 22.7 \mathrm{ng} / \mathrm{ml})$ respect to patients with other autoantibodies $(64.6 \pm 29.7 \mathrm{ng} / \mathrm{ml})$, $\mathrm{p}=0.018$.

Regarding capillaroscopic damage, Chemerin levels were lower in patients with late pattern $(44.8 \pm 18.9 \mathrm{ng} / \mathrm{ml})$ compared to patients with early $(64.3 \pm 28.5 \mathrm{ng} / \mathrm{ml})$ and active pattern $(71.7 \pm 29.9 \mathrm{ng} / \mathrm{ml}), \mathrm{p}<0.001$. APN levels inversely correlate with IL-6 levels $(R=-0.4, p<0.001)$, while directly correlate with capillary density $(\mathrm{R}=0.3, \mathrm{p}=0.03)$. Patients with avascular areas presented lower levels of APN (5.3 $\pm 3.9 \mu \mathrm{g} / \mathrm{ml})$ compared to patients without avascular areas $(7.3 \pm 3.4 \mu \mathrm{g} / \mathrm{ml})$, $\mathrm{p}=0.005$. LEP levels directly correlate with vascular density on nailfold capillaroscopy $(R=0.3, p=0.02)$, confirming the role of LEP in endothelial homeostasis. Furthermore, patients with avascular areas presented lower LEP levels (15.5 $\pm 13.0 \mathrm{ng} / \mathrm{ml}$ ) compared to patients without avascular areas $(31.1 \pm 28.4 \mathrm{ng} / \mathrm{ml})$, $\mathrm{p}=0.003$. LEP levels were lower in patients with active digital ulcers $(9.3 \pm 6.6 \mathrm{ng} /$ $\mathrm{ml})$, compared to patients without ulcers $(9.3 \pm 6.6 \mathrm{ng} / \mathrm{ml}), \mathrm{p}=0.01$. The anti-inflammatory and endothelium protective role of APN emerged also when we considered the lung involvement: in fact patients with DLCO $>50 \%$ presented higher levels of APN $(7.0 \pm 3.9 \mu \mathrm{g} / \mathrm{ml})$ compared to patients with DLCO $<50 \%(5.8$ $\pm 3.8 \mu \mathrm{g} / \mathrm{ml}), \mathrm{p}=0.05$.

Considering the cardiopulmonary involvement, LEP levels inversely correlate with PAPs on echocardiography $(R=-0.24, p=0.02)$. Finally LEP levels inversely correlate with skin score $(\mathrm{R}=-0.3, \mathrm{p}=0.009)$ and patients with early disease presented lower LEP levels $(15.1 \pm 13.2 \mathrm{ng} / \mathrm{ml})$ compared to patients with long lasting disease $(29.9 \pm 28.7 \mathrm{ng} / \mathrm{ml}), \mathrm{p}=0.006$

Conclusions: Our data suggest an imbalance of the levels of adipocytokines in SSc, their down-regulation in patients with a more aggressive pattern on nailfold videocapillaroscopy and organ damage, suggesting a possible role of Chemerin, LEP and APN in the impaired angiogenesis and in the development of vasculopathy of SSc patients.

Disclosure of Interest: None declared

DOI: 10.1136/annrheumdis-2018-eular.7077

\section{OP0108 \\ DIET INDUCED METABOLIC CHANGES IN AN OSTEOARTHRITIS MOUSE MODEL}

M.-L. Hülser ${ }^{1}$, C. Schreiyaeck, Y. Luo ${ }^{2}$, A. Bozec ${ }^{2}$, G. Schett ${ }^{2}$, U. Müller-Ladner', E. Neumann ${ }^{1} .^{1}$ Dept. of Rheumatology and Clinical Immunology, Campus Kerckhoff, Justus-Liebig-University Gießen, Bad Nauheim; ${ }^{2}$ Department Clinic of Medicine 3 - Immunology and Rheumatology, University of Erlangen-Nürnberg, Erlangen, Germany

Background: Adipose tissue is not only an energy depot, but also secrets bioactive factors such as adipokines. Several of these factors are known to regulate immune responses. Osteoarthritis $(\mathrm{OA})$ is one of the most common joint diseases with obesity being a well known risk factor. Therefore, an obesity model (High-fat diet, HFD) was combined with an established model for OA (DMM, destabilisation of the medial meniscus) to evaluate the role of adipokines in this setting

Objectives: This work evaluates the influence of different adipokines on OA progression and obesity comparing systemic vs local effects at different time points. Methods: The study was performed in C57BI/ 6 mice fed with HFD or ND (normal diet) prior to OA induction. Mice were sacrificed and analysed 4, 6 and 8 weeks after surgery. For systemic analysis, sera were evaluated for adipokines such as adiponectin, leptin and visfatin and inflammatory markers. Diet induced systemic changes were also analysed using a fatty liver score and evaluation of crown-like structures (CLS) in adipose tissue. OA progression was scored and quantified based on histological stainings of the joints (H/E, safranin O). Immunohistochemical stainings of the joints were performed to evaluate local distribution of adipokine positive cells and the respective cell types. Metabolic parameters were correlated to the local progression of $O A$

Results: The numbers of CLS were significantly lower comparing HFD (0.2 $\pm 0.1553, \mathrm{n}=7)$ with ND $(5.219 \pm 0.9831, \mathrm{n}=8)$ in mice. Fatty-liver score was significantly higher in HFD compared to ND. OA induction was significant at every timepoint vs healthy control and higher in HFD (e.g.: OA score at 6 weeks HFD 3.7 vs ND 1.4). In the OA groups, there was a positive correlation between bodyweight and $O A$ score $\left(r^{2}=0.33\right)$. Correlation level of $O A$ score with liver score or serum leptin level was low, even though leptin was significantly induced by HFD. However DMM decreased leptin levels at all time points, independent from diet (e.g. 4 weeks: HFD healthy $18.4 \mathrm{ng} / \mathrm{ml}$ vs HFD DMM $3.7 \mathrm{ng} / \mathrm{ml}$ ). The different parameters for metabolic changes (fatty liver score and bodyweight) were positively correlated with serum leptin level. HFD, DMM or the combination of both did not show significant effects on serum levels of adiponectin, visfatin or IL-6. Local adipokine secretion in the joints was independent from systemic metabolism parameters. Conclusions: Our data show that similar to observations in humans, $\mathrm{OA}$ is deteriorated by HFD which correlates mainly with the bodyweight and to a lower extend with metabolic changes induced by obesity. Local adipokine expression was especially detectable in the damaged menisci showing increased amounts of adiponectin and leptin producing cells. Interestingly, local adipokine expression was independent from systemic adipokine levels.

Disclosure of Interest: None declared

DOI: 10.1136/annrheumdis-2018-eular.3082

THURSDAY, 14 JUNE 2018

\section{Biologics in RA. Improving and maintaining the response}

\section{OP0109 IN RA PATIENTS WITH INITIAL RESPONSE TO RITUXIMAB, EARLY DEPTH OF B CELL DEPLETION IS ASSOCIATED WITH LONG TERM MAINTENANCE OF THERAPY}

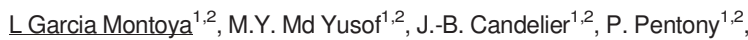
L. Duquenne ${ }^{1,2}$, A.C. Rawstron ${ }^{1,2}$, E.M. Vital ${ }^{1,2}$, P. Emery ${ }^{1,2}$. ${ }^{1}$ Leeds Institute for Rheumatic and Musculoskeletal Medicine, University of Leeds; ${ }^{2}$ NIHR Leeds Biomedical Research Centre, Chapel Allerton Hospital, Leeds, UK

Background: B-cell depletion is a fundamental effect of rituximab (RTX). The speed/depth of initial B-cell depletion is associated with clinical response, and non-responders largely having incomplete depletion. ${ }^{1}{ }^{2}$ However, some patients with incomplete depletion still show clinical improvement (ID-R). Little is known about factors associated with complete depletion; the long-term outcome of the two responder groups according to their level of depletion has not been studied yet.

Objectives: To assess factors that are associated with complete depletion and clinical response (CD-R) and compare the 3 year RTX retention between the two $R T X$ responder groups (CD-R vs ID-R), with a view to inform practice on the optimal use of RTX in RA.

Methods: A prospective observational study was conducted in patients with RA who were treated with RTX in Leeds. Each initial cycle of RTX consisted of $2 \times$ $1000 \mathrm{mg}$ infusions, repeated either on clinical relapse or fixed 6 monthly 